\title{
Circulating tumour cells: insights into tumour heterogeneity
}

\author{
D. F. Hayes \& C. Paoletti \\ From the Breast Oncology Program, University of Michigan Comprehensive Cancer Center, Ann Arbor, MI, USA
}

\begin{abstract}
Hayes DF, Paoletti C (University of Michigan Comprehensive Cancer Center, Ann Arbor, MI, USA). Circulating tumour cells: insights into tumour heterogeneity. (Review). J Intern Med 2013; 274: 137-143.
\end{abstract}

Tumour heterogeneity is a major barrier to cure breast cancer. It can exist between patients with different intrinsic subtypes of breast cancer or within an individual patient with breast cancer. In the latter case, heterogeneity has been observed between different metastatic sites, between metastatic sites and the original primary tumour, and even within a single tumour at either a metastatic or a primary site. Tumour heterogeneity is a function of two separate, although linked, processes. First, genetic instability is a hallmark of malignancy, and results in 'fixed' genetic changes that are almost certainly carried forward through progression of the cancer over time, with increasingly complex additional genetic changes in new metastases as they arise. The second type of heterogeneity is due to differential but 'plastic' expression of various genes important in the biology and response to various therapies. Together, these processes result in highly variable cancers with differential response, and resistance, to both targeted (e.g. endocrine or anti-human epithelial growth receptor type 2 (HER2) agents) and nontargeted therapies (e.g. chemotherapy). Ideally, tumour heterogeneity would be monitored over time, especially in relation to therapeutic strategies. However, biopsies of metastases require invasive and costly procedures, and biopsies of multiple metastases, or serially over time, are impractical. Circulating tumour cells (CTCs) represent a potential surrogate for tissue-based cancer and therefore might provide the opportunity to monitor serial changes in tumour biology. Recent advances have enabled accurate and reliable quantification and molecular characterization of CTCs with regard to a number of important biomarkers including oestrogen receptor alpha and HER2. Preliminary data have demonstrated that expression of these markers between CTCs in individual patients with metastatic breast cancer reflects the heterogeneity of the underlying tumours. Future studies are designed to determine the clinical utility of these novel technologies in either research or routine clinical settings.

Keywords: circulating tumor cells, tumor heterogeneity.

\section{Introduction: tumour heterogeneity}

The concept of tumour heterogeneity in breast cancer has been recognized for decades [1], and interpatient tumour heterogeneity is well established. Malignancies that originate in the breast epithelium have been divided into at least five categories, in addition to the classical histopathological subtypes, based on gene array expression patterns: luminal $\mathrm{A}$ and $\mathrm{B}$, human epithelial growth receptor type 2 (HER2), basal-like and claudin-low [2]. These five subtypes exhibit fundamental biological and untreated characteristics, and treatment is directed by their relative content of oestrogen receptor alpha $(\mathrm{ER} \alpha)$ and HER2 [3, 4].

Of particular relevance, intrapatient heterogeneity has recently received renewed interest as a mech- anism of resistance to systemic therapy [5]. In the late 1970s and 1980s, Goldie and Coldman as well as Norton (with his colleague Simon) generated mathematical models in which they proposed that genetic instability would result in accumulation of mutations and subsequent resistance to chemotherapy during uncontrolled cellular proliferation associated with tumour growth [6, 7]. Skipper and colleagues used rodent models to demonstrate that early tumour transplants were more sensitive to the cytotoxic effects of chemotherapy than tumour grafts that were allowed to grow for longer periods of time before treatment was initiated [8]. These studies were the basis for subsequent prospective randomized clinical trials that established that adjuvant systemic therapy substantially reduces mortality compared with delayed therapy after establishing the presence of metastases [9, 10]. 
Intrapatient heterogeneity also takes several forms. Studies conducted in the 1980 s demonstrated that metastases may have different ER $\alpha$ expression patterns to the original primary cancers from which they arose [11]. These findings have been confirmed and extended to other biomarkers, in particular HER2 [12]. Early findings suggested that this heterogeneity might be a function of treatment pressure [13]; however, subsequent investigations proved that differences in tumour biomarker expression patterns can occur in the absence of any intervening treatment, between different metastases diagnosed simultaneously and even within the same excised primary or metastatic tumour $[11,12,14-17]$.

These findings have several important implications for diagnosis and treatment of patients with breast cancer. First, interpatient tumour heterogeneity may account for the remarkable differences in the risk of a breast cancer recurring in the absence of any systemic therapy (prognosis). Secondly, tumour heterogeneity almost certainly accounts for the problematic issue of resistance to systemic therapies of all types, including chemotherapy and treatments directed against a molecular target such as ER $\alpha$, HER2 and the mammalian target of rapamycin (mTOR) [18]. Although adjuvant systemic treatment is now well established for chemotherapy as well as for endocrine and anti-HER2 agents, at least $15 \%$ of patients with early-stage disease still experience distant metastases, resulting in death in almost all these cases.

It is clear that lack of expression of these targets (i.e. ER $\alpha, \mathrm{HER} 2$ and $\mathrm{mTOR}$ ) renders patients highly unlikely to respond to the specific therapies directed towards them. For example, patients with $\mathrm{ER} \alpha$-negative primary breast cancers do not benefit from adjuvant tamoxifen [9]. However, whereas some of the patients who do express these targets seem to have intrinsic de novo resistance to systemic therapies, many initially show sensitivity but gain 'acquired resistance' over time during treatment. The use of serial single-agent chemotherapies to treat patients with metastatic disease is widespread, providing reasonably high chances of response and benefit but subsequent emergence of resistance to one agent with retained sensitivity to the next, until finally the entire tumour appears to be refractory to all types of treatment [19]. Likewise, it was established more than 40 years ago that administration of serial endocrine therapies to patients with hormone-dependent breast cancers (now defined by expression of $\mathrm{ER} \alpha$-positive cancers) often results in a series of prolonged episodes of response and palliation, each punctuated by subsequent progression, but then response to the next treatment. Unfortunately, as with chemotherapy, ultimately almost all patients develop resistance to endocrine therapy resulting in death due to their disease [20].

The 3rd millennium has ushered in an era of targeted therapy for cancer in general. Breast cancer represents the paradigm of this approach, coupling our understanding of at least two major 'driving' targets, $\mathrm{ER} \alpha$ and HER2, with an astonishing array of specific therapies directed towards them. The recently reported success of adding an mTOR inhibitor, everolimus, to endocrine therapy promises even better outcomes [18], and several other pathways and the agents that target them are now under investigation. Nonetheless, the adverse effects of tumour heterogeneity will remain a critical issue for the use of these therapies in a systematic manner.

\section{Mechanisms of tumour heterogeneity}

In a landmark paper published about 30 years ago, Fidler and Hart first demonstrated emergence of intratumour heterogeneity in murine models [21]. More recent studies, using next-generation genomic technologies, have provided insight into the overwhelming genetic diversity between different breast cancers [22-24] and between primary and metastatic tumours in the same patient [25]. Demonstration in a patient with renal cell cancer of the evolution of genetic heterogeneity between and amongst different metastatic tumours has confirmed that accumulation of mutations and other genetic variations (amplifications and deletions) results in increasingly complex, although still viable, clones that ultimately lead to mortality [5]. Indeed, this cumulative complexity has been implicated as at least one mechanism of resistance to aromatase inhibition in ER $\alpha$-positive breast cancer [24].

Genetic heterogeneity due to chromosomal instability is a relatively fixed phenomenon. In other words, once a clone has a genetic variation, this variation is likely to remain constant throughout the evolution of that clone, even as it acquires further genetic variations. Although it is possible that a mutation might be eliminated by back mutation (i.e. reversion to wild type), this appears to occur rarely, and a mutation to a cellular lethal event, eliminating the clone altogether, is more likely. 
However, in addition to fixed tumour heterogeneity, 'plastic' heterogeneity must also be considered. In this case, for a variety of reasons, expression of a driving protein pathway is not under control of a fixed constitutive mechanism, but rather is subject to environmental stresses resulting in up- or downregulation. Such regulation may be due to interpathway cross-talk. For example, it has been demonstrated in several preclinical studies that apparently ER $\alpha$-positive, 'HER2-negative' cancers can in fact upregulate HER2 in the presence of anti-oestrogenic agents [26-29]. Thus, to guide treatment strategies, clinical scientists and caregivers will need to be able to monitor both the emergence of fixed heterogeneity within a patient and treatment-related plastic tumour variability.

\section{Clinical implications of tumour heterogeneity: potential role of circulating tumour cells}

The phenomenon of emerging variable tumour heterogeneity must be taken into consideration as therapeutic trials of new targeted therapies are being planned. It is highly probable that each new targeted therapy introduced in the clinic will induce transient, short-term benefit to which the tumour will ultimately become resistant. Although such responses represent steps forward, they are unlikely to result in substantial gains in overall survival or increases in cure rates.

By implication, using the tissue collected from a patient's primary cancer to predict the best treatment many years later, after several prior therapies, is unlikely to provide reliable information. Likewise, monitoring accumulated genetic or plastic changes in cancers, as the patient is treated, might guide selection of the next therapy, or even addition of a subsequent therapy, whilst maintaining the treatment on which the cancer has already progressed. Ideally, these concerns would be resolved by performing a biopsy of the metastases at each critical clinical stage. A biopsy to establish the diagnosis and determine whether the predictive biomarkers of metastases remain concordant, or have changed, compared with the primary cancer, is now fairly well established as the standard of care. However, most metastases are localized in internal organs (liver, lung and bone), and therefore, biopsies are invasive, inconvenient and costly, and multiple biopsies are logistically difficult if not impossible.

In this regard, detection and characterization of circulating tumour cells (CTCs) might serve as a 'liquid' real-time biopsy. CTCs were first identified postmortem by Ashworth nearly 150 years ago [30]. However, until recently, accurate and reliable methods of isolating, quantifying and genotyping or phenotyping CTCs were not available. Over the last decade, a number of strategies to isolate CTCs from the billions of erythrocytes and millions of leucocytes present in a single blood sample have been investigated [31]. These approaches are based on the physical differences between cancer and normal haematopoietic cells, such as size or expression of either epithelial-specific or relatively cancer-specific molecules, such as epithelial cell adhesion molecule (EpCAM) or HER2.

Of these, the most widely used commercially available assay is the CellSearch ${ }^{\circledR}$ system (Veridex,

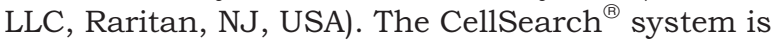
a highly automated assay based on an anti-EpCAM immunomagnetic capture step followed by fluorescent images that capture nucleated (DAPI-positive), cytokeratin-positive CD45-negative events that are designated as CTCs (Fig. 1). It has recently been demonstrated in several studies in patients with metastatic breast, colorectal and prostate cancer, as well as in the adjuvant breast cancer setting, that those who have elevated baseline CTC levels, determined using CellSearch ${ }^{\circledR}$, have worse prognosis than those with normal levels [31-33].

However, as these studies are plagued by obstacles to all diagnostic approaches, sensitivity and specificity, only approximately $50 \%$ of patients with metastatic breast cancer have elevated CTC levels at baseline (defined as $\geq 5$ CTCs $/ 7.5 \mathrm{~mL}$ whole blood), and in the adjuvant setting, only approximately 10-25\% have $\geq 1 \mathrm{CTC} / 7.5-23 \mathrm{~mL}$ whole blood [31, 33, 34]. Furthermore, even if CTC levels are elevated using these definitions, very few patients have more than $10 \mathrm{CTCs} / 7.5 \mathrm{~mL}$ whole blood, which severely limits the ability to further characterize the CTC in any meaningful manner. This lack of sensitivity may be due to both technical and biological factors. In any assay, repeated handling and washing of a specimen will result in loss of analyte. Moreover, because the CellSearch ${ }^{\circledR}$ system is based on EpCAM expression, cancers that do not express this marker (estimated at about $20 \%$ ), or individual cells within tumours that do not express EpCAM, may not be captured [35].

Similarly, specificity may be a technical or biological issue. An assay that increases sensitivity is more prone to capturing nonmalignant cells, 


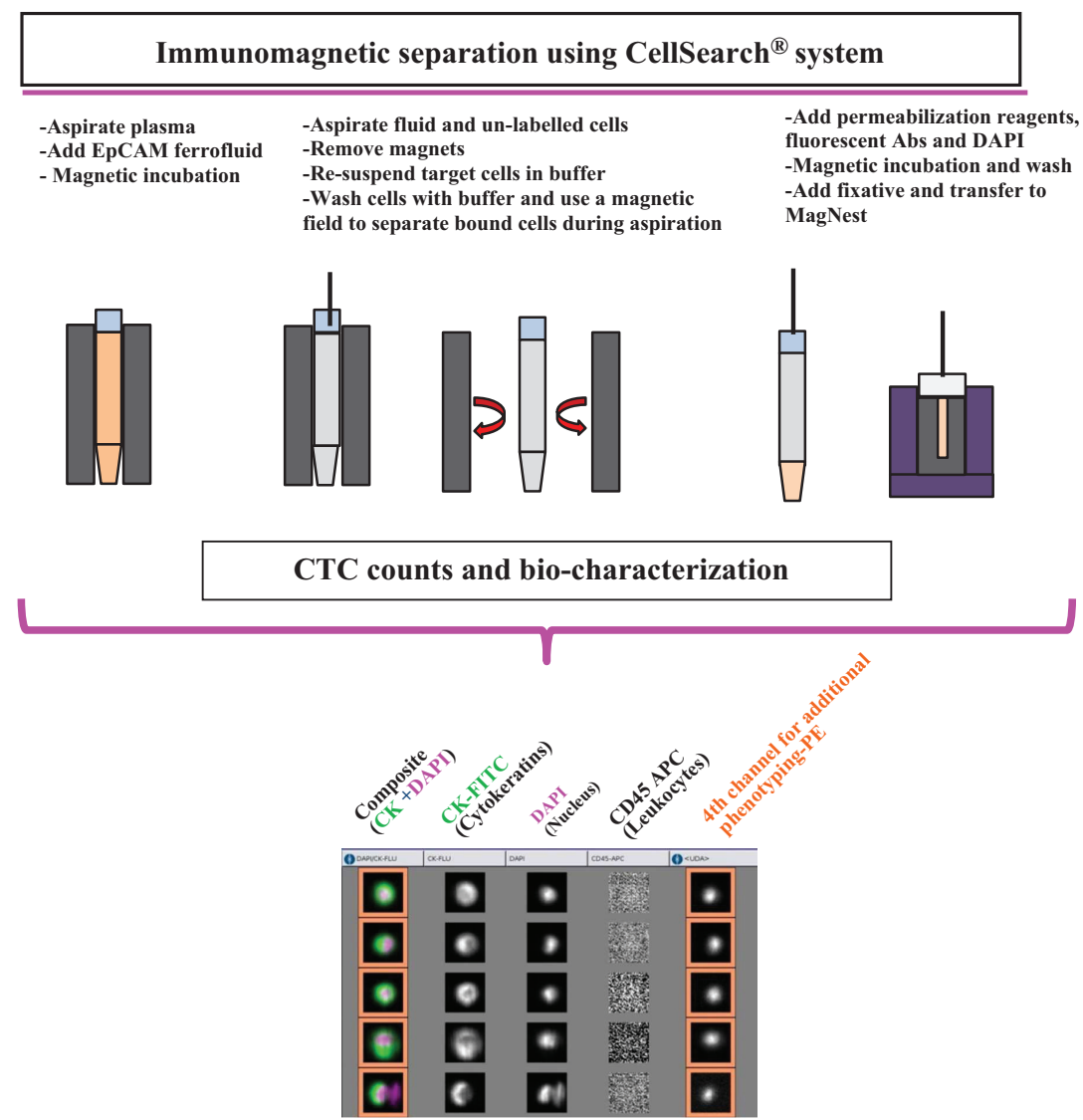

Fig. 1 Immunomagnetic separation and characterization of circulating tumour cells (CTCs) using the CellSearch ${ }^{\circledR}$ system. CTCs are isolated from whole blood using anti-EpCAM-coated nanomagnetic particles and then characterized for the presence of a nucleus (by DAPI staining) and expression of cytokeratin (FITC-labelled anticytokeratin). Demonstration that the cells are not leucocytes is determined by lack of staining with APC-labelled CD45. The fluorescent 'events' are captured by automatic fluorescent laser microscopy and presented in separate channels (labelled, respectively) and as a composite channel. A separate fourth channel is available for characterization of other markers.

emphasizing the importance of the second characterization step in CellSearch ${ }^{\circledR}$ and other emerging assays. Perhaps of more importance, even if a captured cell is considered morphologically or phenotypically to represent a cancer cell, it may not have malignant potential. It has been demonstrated in several clinical studies that the presence of microscopic malignant cells at sites external to the primary tumour (lymph nodes, bone marrow or the circulation) is associated with poor prognosis $[33,36,37]$. However, the presence of documented micrometastases does not imply absolute risk of subsequent recurrence. Indeed, Meng et al. [38] reported that 13 of $36(36 \%)$ women who had no evidence of clinical disease 7-22 years after mastectomy had detectable aneusomic CTCs. Furthermore, Weidswang et al. reported that 53 of 356
$(15 \%)$ patients who were disease-free after 3 years of follow-up had bone marrow micrometastases. After a subsequent follow-up period of approximately 3 years, only $21 \%$ of these patients with documented persistent bone marrow metastases relapsed [39]. Taken together, these studies suggest that simply finding more cells using highsensitivity assays may not have clinical implications and that future studies using next-generation capture devices need to be planned carefully, taking into consideration clinical outcomes and not just diagnostic comparisons with the current gold standard.

Nonetheless, molecular characterization of captured CTCs might provide insight into the future clinical behaviour of the cancer, especially in 
relation to targeted therapy. However, it is not clear that CTCs actually reflect the biology of the tissuebased cancer. Rather, it is possible that the detected cells with currently available techniques are merely those that were shed and are only the 'tip of the iceberg'. These cells do not necessarily represent the biology of the underlying tumour. For example, it is clear that an elevated number of CTCs as detected by CellSearch ${ }^{\circledR}$ are associated with poor prognosis, but one does not know whether these cells actually have malignant behaviour themselves. Perhaps they are terminally differentiated cells that simply reflect the presence of more malignant, but uncaptured, cancer stem cells [40]. Recent observations that cancer cells undergo epithelial to mesenchymal transformation, with loss of EpCAM, as a critical part of the metastatic process further suggest that the truly malignant cell might not be detected with currently available assays [41, 42].

A number of groups have now demonstrated the technical ability to perform molecular characterization of CTCs. Protein biomarkers such as ER $\alpha$, HER2, Ki67, BCL2 androgen receptor, and insulinlike growth factor, as well as HER2 amplification and multi-gene transcript expression, can be measured reliably and accurately [31, 43-47]. Furthermore, using other platforms, the ability to detect these and other markers [48, 49] as well as DNA variations such as mutations in EGFR [50] have been demonstrated. However, at present, there is no evidence that the biomarker status of a CTC predicts clinical response or outcome; indeed, it was recently reported that HER2 positivity in CTCs was not associated with response to lapatinib [51].

Regardless of the immediate biological or clinical applications of CTCs, these studies have demonstrated that, like their associated cancers, these cells are remarkably heterogeneous [52]. Expression levels of HER2 in CTCs can vary considerably within individual patients [53] (Fig. 2). Using the CellSearch $^{\circledR}$ system, we recently developed a CTCEndocrine Therapy Index, based on semi-quantitative phenotyping of CTCs for ER $\alpha$, BCL2, HER2 and Ki67. In a pilot study, we rarely observed a single patient in whom all of the CTCs expressed any one of these markers uniformly [43]. Rather, there was generally a broad range of expression of each marker in the CTCs identified in individuals with metastatic breast cancer. We speculate that CTC heterogeneity might provide a more biologically and clinically useful description of the heter-
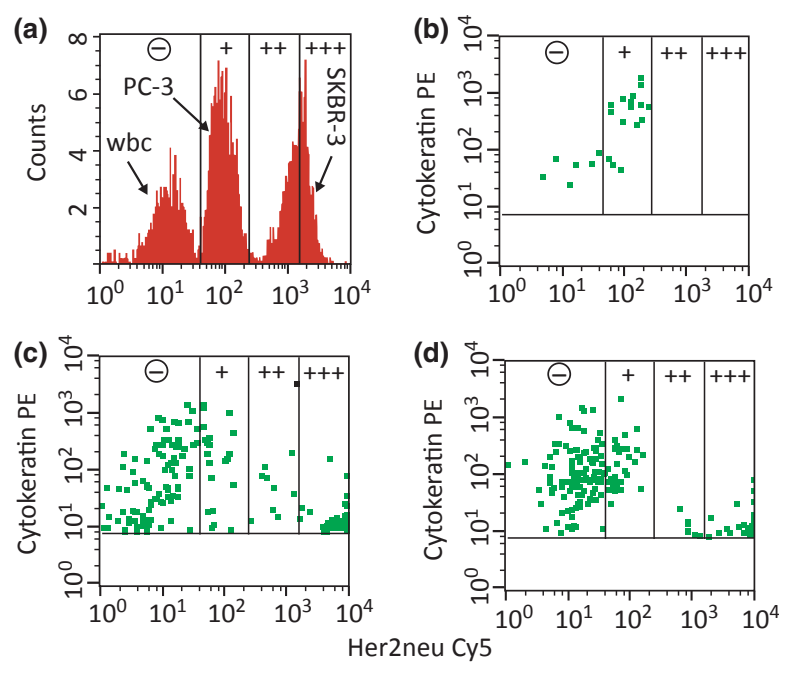

Fig. 2 Heterogeneity of human epithelial growth receptor type 2 (HER2) expression in cell lines and on circulating tumour cells (CTCs) from three patients with breast cancer. (a) HER2 expression on leucocytes, PC3 cells and SkBr-3 cells immunomagnetically selected from $5 \mathrm{~mL}$ blood and gated on CD45 and cytokeratin expression. The expression levels of HER2 were subdivided into four categories $(-,+,++,+++)$ based on the quantitative assessment of HER 2 expression on $\mathrm{PC} 3$ and $\mathrm{SkBr}-3$ cells. -, no expression below 5000 receptors (i.e. wbcs); +, expression of between 5000 and 50000 receptors (PC-3); ++, expression of between 50000 and 500000 receptors; +++, expression of more than 500000 receptors ( $\mathrm{SkBr}-3)$. ( $b-d)$, expression of cytokeratin and HER2 on CTCs from three patients with breast cancer. Adapted from Hayes et al. [53] with permission.

ogeneity of the entire tumour burden within a patient, compared with biopsies of individual metastatic lesions. However, it is also possible, as discussed above, that the molecular features of CTCs may not be representative of the tissue-based tumour behaviour. Future studies are planned to investigate the clinical implications of the molecular characteristics of CTCs, the heterogeneity of these characteristics and the changes over time during treatment.

\section{Conclusions}

In summary, the biological implications of tumour heterogeneity, which has been recognized for years, are fundamental to therapeutic resistance and therefore mortality due to breast cancer. CTCs may offer a virtual, or 'real-time', opportunity to perform 'liquid biopsies', thus permitting a more 
accurate prediction of the likelihood of response to specific therapies and greater insight into the mechanisms of emerging resistance during treatment.

\section{Conflict of interest statement}

Dr. Hayes has received research support from Veridex, LLC, the manufacturer of the CellSearch ${ }^{\circledR}$ system. Dr. Hayes has a patent application pending for the use of CTC-ETI.

\section{Acknowledgements}

This work was supported in part by Fashion Footwear Charitable Foundation of New York/ QVC Presents Shoes on Sale ${ }^{\mathrm{TM}}$ (DFH and CP) and the Associazione Sandro Pitigliani (CP) and by a studentship from the Italian Foundation for Cancer Research (CP).

\section{References}

1 Schnipper L. Clinical implications of tumor-cell heterogeneity. $N$ Engl J Med 1986; 314: 1423-32.

2 Perou CM, Sorlie T, Eisen MB et al. Molecular portraits of human breast tumours. Nature 2000; 406: 747-52.

3 Smith IE, Dowsett M. Aromatase inhibitors in breast cancer. $N$ Engl $J$ Med 2003; 348: 2431-42.

4 Hortobagyi GN. Trastuzumab in the treatment of breast cancer. $N$ Engl J Med 2005; 353: 1734-6.

5 Gerlinger M, Rowan AJ, Horswell S et al. Intratumor heterogeneity and branched evolution revealed by multiregion sequencing. N Engl J Med 2012; 366: 883-92.

6 Goldie JH, Coldman AJ. A mathematic model for relating the drug sensitivity of tumors to their spontaneous mutation rate. Cancer Treat Rep 1979; 63: 1727-33.

7 Norton L. Implications of kinetic heterogeneity in clinical oncology. Semin Oncol 1985; 12: 231-49.

8 Skipper HE, Schabel FM, Wilcox WS. Experimental evaluation of potential anticancer agents. XII. On the criteria and kinetics associated with curability of experimental leukemia. Cancer Chemother Rep 1964; 35: 1-111.

9 Early Breast Cancer Trialists' Collaborative Group. Effects of chemotherapy and hormonal therapy for early breast cancer on recurrence and 15-year survival: an overview of the randomised trials. Lancet 2005; 365: 1687-717.

10 Early Breast Cancer Trialists Collaborative Group T. Comparisons between different polychemotherapy regimens for early breast cancer: meta-analyses of long-term outcome among 100,000 women in 123 randomized trials. Lancet 2012; 379: 432-44.

11 Hull DF 3rd, Clark GM, Osborne CK, Chamness GC, Knight WA 3rd, McGuire WL. Multiple estrogen receptor assays in human breast cancer. Cancer Res 1983; 43: 413-6.

12 Lindstrom LS, Karlsson E, Wilking UM et al. Clinically used breast cancer markers such as estrogen receptor, progester- one receptor, and human epidermal growth factor receptor 2 are unstable throughout tumor progression. $J$ Clin Oncol 2012; 30: 2601-8.

13 Allegra JC, Barlock A, Huff KK, Lippman ME. Changes in multiple or sequential estrogen receptor determinations in breast cancer. Cancer 1980; 45: 792-4.

$14 \mathrm{Wu}$ JM, Fackler MJ, Halushka MK et al. Heterogeneity of breast cancer metastases: comparison of therapeutic target expression and promoter methylation between primary tumors and their multifocal metastases. Clin Cancer Res 2008; 14: 1938-46.

15 Wicha MS. Cancer stem cell heterogeneity in hereditary breast cancer. Breast Cancer Res 2008; 10: 105.

16 Cardoso F, Di Leo A, Larsimont D et al. Evaluation of HER2, p53, bcl-2, topoisomerase II-alpha, heat shock proteins 27 and 70 in primary breast cancer and metastatic ipsilateral axillary lymph nodes. Ann Oncol 2001; 12: 615-20.

17 Guidi AJ, Berry DA, Broadwater G et al. Association of angiogenesis in lymph node metastases with outcome of breast cancer. J Natl Cancer Inst 2000; 92: 486-92.

18 Baselga J, Campone M, Piccart $\mathrm{M}$ et al. Everolimus in postmenopausal hormone-receptor-positive advanced breast cancer. N Engl J Med 2012; 366: 520-9.

19 Ellis M, Hayes DF, Lippman ME. Treatment of metastatic breast cancer. In: Harris J, Lippman M, Morrow M, Osborne $\mathrm{CK}$, eds. Diseases of the Breast. Philadelphia: Lippincott Williams \& Wilkins, 2004; 1101-62.

20 Kennedy BJ. Endocrine therapy of breast cancer. $J$ Am Med Assoc 1967; 200: 971-2.

21 Fidler IJ, Hart IR. Biological diversity in metastatic neoplasms: origins and implications. Science 1982; 217: 998 1003.

22 Curtis C, Shah S, Chin S-F et al. The genomic and transcriptomic architecture of 2,000 breast tumors reveals novel subgroups. Nature 2012; 486: 346-52.

23 Shah SP, Roth A, Goya R et al. The clonal and mutational evolution spectrum of primary triple-negative breast cancers. Nature 2012; 486: 395-9.

24 Ellis MJ, Ding L, Shen D et al. Whole-genome analysis informs breast cancer response to aromatase inhibition. Nature 2012; 486: 353-60.

25 Ding L, Ellis MJ, Li S et al. Genome remodelling in a basallike breast cancer metastasis and xenograft. Nature 2010; 464: 999-1005.

26 Bayliss J, Hilger A, Vishnu P, Diehl K, El-Ashry D. Reversal of the estrogen receptor negative phenotype in breast cancer and restoration of antiestrogen response. Clin Cancer Res 2007; 13: 7029-36.

27 Gee JM, Robertson JF, Gutteridge E et al. Epidermal growth factor receptor/HER2/insulin-like growth factor receptor signalling and oestrogen receptor activity in clinical breast cancer. Endocr Relat Cancer 2005; 12(Suppl. 1): S99-111.

28 Hayes E, Nicholson RI, Hiscox S. Acquired endocrine resistance in breast cancer: implications for tumour metastasis. Front Biosci 2011; 16: 838-48.

29 Gilani RA, Kazi AA, Shah P et al. The importance of HER2 signaling in the tumor-initiating cell population in aromatase inhibitor-resistant breast cancer. Breast Cancer Res Treat 2012; 135: 681-92.

30 Ashworth TR. A case of cancer in which cells similar to those in the tumours were seen in the blood after death. Aust Med $J$ 1869; 14: 146-9. 
31 Paoletti C, Smerage J, Hayes DF. Circulating tumor cells as a marker of prognosis. Princip Prac Oncol 2012; 26: 1-8.

32 Rack B, Schindlbeck C, Andergassen U et al. Use of circulating tumor cells in peripheral blood of breast cancer patients before and after adjuvant chemotehapy to predict increased risk for relapse: the SUCCESS trial. Proc Am Soc Clin Oncol 2010; 28: 114s (Abstract \#1003).

33 Lucci A, Hall CS, Lodhi AK et al. Circulating tumour cells in non-metastatic breast cancer: a prospective study. Lancet Oncol 2012; 13: 688-95.

34 Rack B, Schindlbeck C, Andergassen U et al. Prognostic role of circulating tumor cells in the peripheral blood of primary breast cancer patients. Cancer Res 2010; 24(supp1): 93 (abstract S6-5).

35 Hayes DF, Cristofanilli M. Re: anti-epithelial cell adhesion molecule antibodies and the detection of circulating normallike breast tumor cells. J Natl Cancer Inst 2009; 101: 894-5 author reply 6-7.

36 Haagensen CD. Diseases of the Breast. Philadelphia: W.B. Saunders, 1971.

37 Braun S, Marth C. Circulating tumor cells in metastatic breast cancer - toward individualized treatment? $N$ Engl $J$ Med 2004; 351: 824-6.

38 Meng S, Tripathy D, Frenkel EP et al. Circulating tumor cells in patients with breast cancer dormancy. Clin Cancer Res 2004; 10: 8152-62.

39 Wiedswang G, Borgen E, Karesen R et al. Isolated tumor cells in bone marrow three years after diagnosis in disease-free breast cancer patients predict unfavorable clinical outcome. Clin Cancer Res 2004; 10: 5342-8.

40 Wicha MS,Hayes DF. Circulating tumor cells: not all detected cells are bad and not all bad cells are detected. $J$ Clin Oncol 2011; 29: 1508-11.

41 Kalluri R. EMT: when epithelial cells decide to become mesenchymal-like cells. J Clin Invest 2009; 119: 1417-9.

42 Kalluri R, Weinberg RA. The basics of epithelial-mesenchymal transition. J Clin Invest 2009; 119: 1420-8.

43 Paoletti C, Connelly M, Chianese D et al. Development of Circulating Tumor Cell-Endocrine Therapy Index in metastatic breast cancer patients. Clinical Cancer Research Proceedings of the San Antonio Breast Cancer Symposium 2011; 24(supp1): 451s (abst. p4-07-016).
44 Neubauer H, Clare SE, Wozny W et al. Breast cancer proteomics reveals correlation between estrogen receptor status and differential phosphorylation of PGRMC1. Breast Cancer Res 2008; 10: R85.

45 Fehm T, Krawczyk N, Solomayer EF et al. ERalpha-status of disseminated tumour cells in bone marrow of primary breast cancer patients. Breast Cancer Res 2008; 10: R76.

$46 \mathrm{Fehm} \mathrm{T,} \mathrm{Becker} \mathrm{S,} \mathrm{Duerr-Stoerzer} \mathrm{S} \mathrm{et} \mathrm{al.} \mathrm{Determination} \mathrm{of}$ HER2 status using both serum HER2 levels and circulating tumor cells in patients with recurrent breast cancer whose primary tumor was HER2 negative or of unknown HER2 status. Breast Cancer Res 2007; 9: R74.

47 Danila DC, Fleisher M, Scher HI. Circulating tumor cells as biomarkers in prostate cancer. Clin Cancer Res 2011; 17: 3903-12.

48 Pecot CV, Bischoff FZ, Mayer JA et al. A novel platform for detection of CK+ and CK- CTCs. Cancer Discov 2011; 1: 580-6.

49 Powell AA, Talasaz AH, Zhang $\mathrm{H}$ et al. Single cell profiling of circulating tumor cells: transcriptional heterogeneity and diversity from breast cancer cell lines. PLOS ONE 2012; 7: e33788.

50 Maheswaran S, Sequist LV, Nagrath $\mathrm{S}$ et al. Detection of mutations in EGFR in circulating lung-cancer cells. $N$ Engl J Med 2008; 359: 366-77.

51 Pestrin M, Bessi S, Puglisi $\mathrm{F}$ et al. Final results of a multicenter phase II clinical trial evaluating the activity of single-agent lapatinib in patients with HER2-negative metastatic breast cancer and HER2-positive circulating tumor cells. A proof-of-concept study. Breast Cancer Res Treat 2012; 134: $283-9$

52 Armstrong AJ, Eisenberger MA, Halabi S et al. Biomarkers in the management and treatment of men with metastatic castration-resistant prostate cancer. Eur Urol 2012; 61: 549-59.

53 Hayes DF, Walker TM, Singh B et al. Monitoring expression of HER-2 on circulating epithelial cells in patients with advanced breast cancer. Int $J$ Oncol 2002; 21: 1111-7.

Correspondence: Daniel F. Hayes, MD, 6312 Cancer Center, University of Michigan 1500 E. Medical Center Dr., Ann Arbor, MI 48109-5942, USA.

(fax: 734-647-9271; e-mail: hayesdf@umich.edu) 